\title{
Plasmablastic lymphoma: one or more tumors?
}

\section{Stefano A. Pileri, ${ }^{1}$ Saveria Mazzara ${ }^{1}$ and Enrico Derenzini ${ }^{2}$}

${ }^{1}$ Division of Hematopathology, European Institute of Oncology IRCCS and ${ }^{2}$ Division of Hemato-oncology, European Institute of Oncology IRCCS, Milan, Italy

E-mail: STEFANO A. PILERI - stefano.pileri@ieo.it

doi:10.3324/haematol.2021.278841

\begin{abstract}
T $\mathrm{n}$ this issue of Haematologica, Ramis-Zaldivar et al. report on a series of cases of plasmablastic lymphoma L(PBL), studied by a high-resolution approach to unravel the genomic landscape of the tumor, which has remained so far largely unexplored. ${ }^{1}$ According to the revised fourth edition of the World Health Organization (WHO) Classification of Tumours of Haematopoietic and Lymphoid Tissues, PBL is a rare and very aggressive disease entity with diffuse proliferation of large neoplastic cells, most of which resemble B immunoblasts or plasmablasts that have a CD20-negative plasmacytic phenotype and frequently carry MYC rearrangements. ${ }^{2}$ PBL was originally described in the oral cavity and frequently occurs in association with human immunodeficiency virus (HIV) infection, but it may also develop at other sites, predominantly extranodal, and in association with other causes of immunodeficiency. ${ }^{2}$ In the majority of cases, neoplastic cells are infected by Epstein-Barr virus (EBV). ${ }^{2}$ The differential diagnosis may include other varieties of diffuse large B-cell lymphoma, Burkitt lymphoma, plasmablastic plasma cell myeloma, and anaplastic large cell lymphoma. There is no standard of care at the time being and immunotherapy with antiCD20 antibodies is unfeasible because of the lack of the target. DA-EPOCH chemotherapy has been recom-
\end{abstract}

mended, possibly in conjunction with combined antiretroviral therapy in HIV-positive patients. ${ }^{3}$ However, studies of patients with PBL treated with chemotherapy regimens more intensive than $\mathrm{CHOP}$ did not identify a survival benefit and anyway the median overall survival with DA-EPOCH treatment is disappointing, being reported as 15 months in HIV-positive patients, and likely worse in HIV-negative patients. ${ }^{4}$ The usage of other therapies, such as chimeric antigen receptor T-cell therapy, remains anecdotal, ${ }^{5}$ the optimal treatment of PBL being still an open issue.

Ramis-Zaldivar et al. applied a highly refined approach to 34 cases of PBL; their investigations included mutational and copy number analyses, targeted gene expression profiling, fluorescence in situ hybridization, and immunohistochemistry. ${ }^{1}$ Such an array of techniques is the ideal way to explore the pathobiology of each neoplasm, if one aims to understand the possible range of mechanisms which sustain it, and discover novel potential therapeutic targets.

Their study highlights the molecular heterogeneity of $\mathrm{PBL}$, which is in keeping with the scenario arising in the field of malignant lymphomas in general. For a long time, lymphomas have been considered monoclonal/ monolithic conditions. It is now becoming increasingly

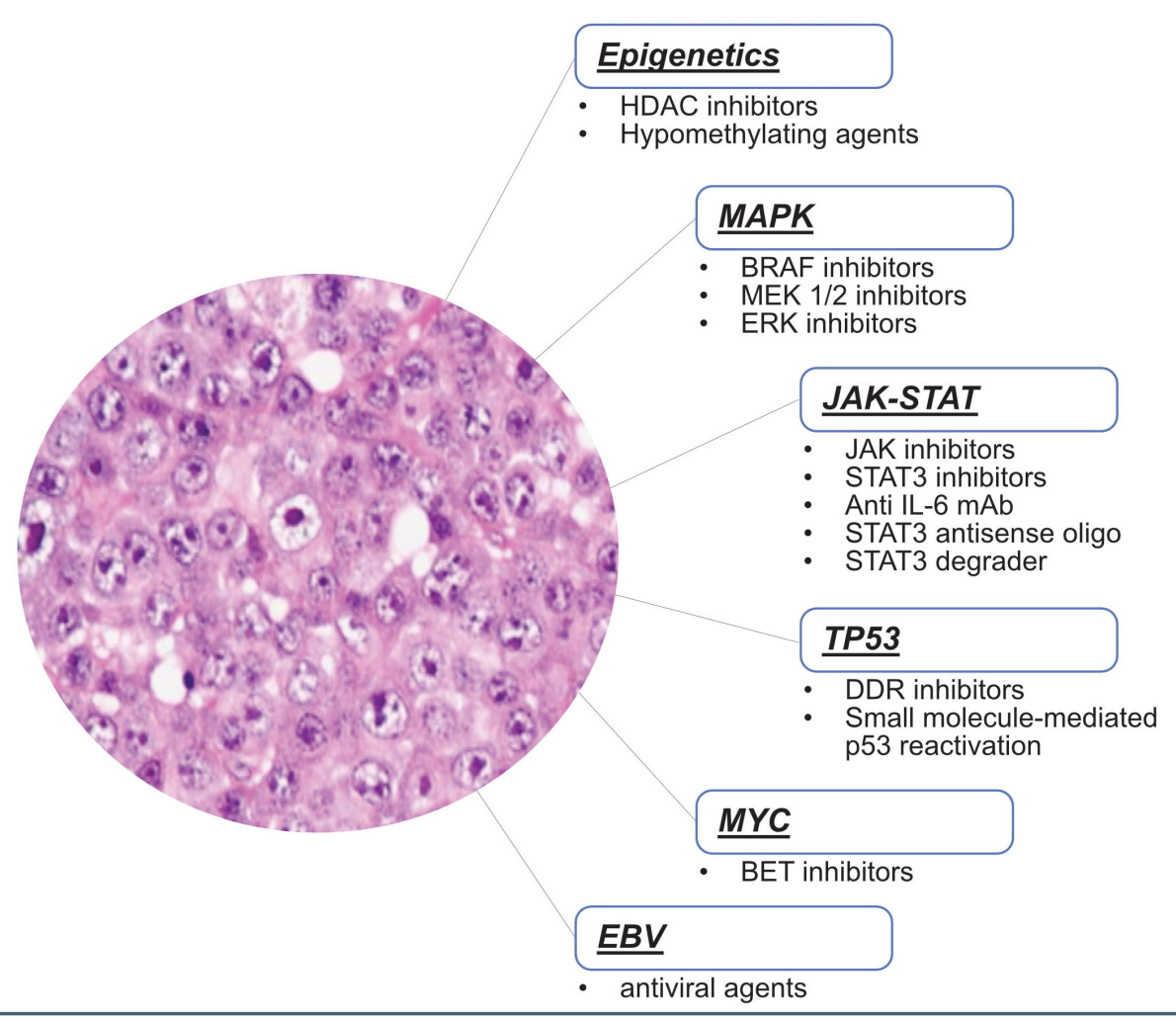

Figure 1. Array of potential targeted therapies based on specific molecular abnormalities detected in plasmablastic lymphomas. 
evident that the same morphological and phenotypic features can be sustained by a quite variable landscape of genetic aberrations with clones competing with each other within the same neoplasm and under the influence of the microenvironment, thus leading to possible clonal selection, chemoresistance and relapse. ${ }^{6}$

Ramis-Zaldivar et al. show that PBL is characterized by high genetic complexity with very frequent $M Y C$ translocations (87\%), gains of 1q21.1-q44, trisomy 7 , 8q23.2-q24.21, 11p13-p11.2, 11q14.2-q25, 12p and 19p13.3-p13.13, losses of $1 \mathrm{p} 33,1 \mathrm{p} 31.1-\mathrm{p} 22.3,13 \mathrm{q}$ and 17p13.3-p11.2, and recurrent mutations of STAT3, NRAS, TP53, MYC, EP300, CARD11, SOCS1, and TET2 (here listed in order of decreasing frequency). ${ }^{1}$ Pathway enrichment analysis suggested a cooperative action between MYC alterations and MAPK and JAK-STAT signaling pathways. These alterations have been at least in part reported in previous studies. ${ }^{7.8}$ However, RamisZaldivar et al. achieved three major goals. First, they analyzed the clonal evolution in PBL and found that in 24 tumors the cancer cell fraction included 250 copy number alterations and 107 mutations. Most of these alterations revealed a wide spectrum of cancer cell fractions with the exception of TP53 mutations, 17 losses and $13 \mathrm{q}$ deletions, which turned out to be clonal. Notably, clonal and subclonal mutations affected the same protein domains, suggesting that the subclonal ones give similar advantage to the neoplastic cell. This is in keeping with what has been observed in liquid biopsy studies, which have revealed that the mutational landscape of diffuse large B-cell lymphoma is definitely wider in circulating tumoral DNA than in the diagnostic biopsy. ${ }^{9}$ This finding underlines the potential occurrence of different clones at different anatomic sites (clonal heterogeneity) in lymphoid malignancies characterized by high genetic complexity, clones which can be selected by therapy and cause disease resistance or relapse. ${ }^{10}$ Second, the recorded constellation of genetic alterations could represent the rationale for targeted therapies (Figure 1), which are indeed urgently needed in the light of the poor response to conventional chemotherapy, including intensified regimens. The third important achievement of the study by Ramis-Zaldivar et al. consists in the demonstration of a clear-cut genetic difference between EBV-positive and EBV-negative cases of PBL. In particular, the latter showed greater genetic complexity and higher mutational loads than the EBVpositive ones. EBV-negative cases were characterized by more frequent mutations affecting TP53, CARD11 and MYC as well as epigenome/chromatin modifiers, cell cycle and the NF-кB pathway. Conversely, EBV-positive cases tended to carry frequent mutations involving genes of the JAK-STAT pathway. These findings are of interest for practical and conceptual reasons. They suggest different therapeutic targets depending on EBVpositivity or negativity. Furthermore, they strengthen the understanding of the different pathobiology of virus-infected lymphomas. By RNA sequencing, Abate et al. showed that eight (40\%) of 20 cases of endemic Burkitt lymphoma from Uganda carried infection by Herpesviridae members other than EBV, thus suggesting a polyviral condition, and revealed a mutational land- scape different from that of sporadic Burkitt lymphoma (with lower frequencies of MYC, ID3, TCF3 and TP53 mutations, higher frequency of ARID1A mutations, and the occurrence of not previously detected $R H O A$ and CCNF mutations). ${ }^{11}$ These findings suggested, as in the report from Ramis-Zaldivar et al., ${ }^{1}$ a dual mechanism of transformation in epidemic and sporadic Burkitt lymphoma: virus versus mutation-driven, respectively.

The ever increasing application of high-resolution approaches to the analysis of malignant lymphomas is unraveling a scenario much more intriguing than thought only a few years ago. Within the body of a distinct entity as defined by the WHO classification, ${ }^{2}$ there are tumors with different pathobiological characteristics, which offer different targets for ad hoc therapies. This has practical implications for the management of patients as well as for the design of innovative therapeutic trials, if the final goal is to move to precision medicine.

\section{Disclosures}

No conflicts of interest to disclose.

\section{Contributions}

SAP wrote the manuscript; ED commented and revised it; $S M$ created the figure.

\section{Funding}

Supported by the grant AIRC $5 \times 1000$ n. 21198.

\section{References}

1. Ramis-Zaldivar JE, Gonzales-Farre B, Nicolae A, et al. MAPK and JAK-STAT pathways dysregulation in plasmablastic lymphoma. Haematologica. 2021;106(10):2682-2693.

2. Swerdlow SH, Campo E, Harris NL, et al. WHO Classification of Tumour of Haematopoietic and Lymphoid Tissues, Revised 4th edition. 2017. IARC Press, Lyon.

3. Castillo JJ, Bibas M, Miranda RN. The biology and treatment of plasmablastic lymphoma. Blood. 2015;125(15):2323-2330.

4. Castillo JJ, Furman M, Beltrán BE, et al. Human immunodeficiency virus-associated plasmablastic lymphoma: poor prognosis in the era of highly active antiretroviral therapy. Cancer. 2012;118(21): 5270-5277.

5. Raychaudhuri R, Qualtieri J, Garfall AL. Axicabtagene ciloleucel for CD19+ plasmablastic lymphoma. Am J Hematol. 2020;95(1): E28-E30.

6. Kotlov N, Bagaev A, Revuelta MV, et al. Clinical and biological subtypes of B-cell lymphoma revealed by microenvironmental signatures. Cancer Discov. 2021;11(6):1468-1489.

7. Garcia Reyero J, Martinez Magunacelaya N, Gonzalez de Villambrosia S, et al. Genetic lesions in MYC and STAT3 drive oncogenic transcription factor overexpression in plasmablastic lymphoma. Haematologica. 2021;106(4): 1120-1128.

8. Liu Z, Filip I, Gomez K, et al. Genomic characterization of HIVassociated plasmablastic lymphoma identifies pervasive mutations in the JAK-STAT pathway. Blood Cancer Discov. 2020;1(1):112125 .

9. Kurtz DM, Scherer F, Jin MC, et al. Circulating tumor DNA measurements as early outcome predictors in diffuse large B-cell lymphoma. J Clin Oncol. 2018;36(28):2845-2853.

10. Derenzini E, Iacobucci I, Agostinelli C, et al. Therapeutic implications of intratumor heterogeneity for TP53 mutational status in Burkitt lymphoma. Exp Hematol Oncol. 2015;4:24.

11. Abate F, Ambrosio MR, Mundo L, et al. Distinct viral and mutational spectrum of endemic Burkitt lymphoma. Plos Pathogens. 2015;11(10):e1005158. 